\title{
Crizotinib in Chinese Patients with ROS1-Rearranged Advanced Non- Small-Cell Lung Cancer in Routine Clinical Practice
}

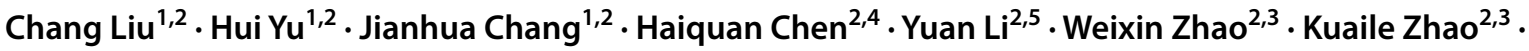 \\ Zhengfei Zhu ${ }^{2,3} \cdot$ Si Sun $^{1,2} \cdot$ Min Fan $^{2,3} \cdot$ Jialei Wang ${ }^{1,2}$ (1)
}

Published online: 11 April 2019

(c) The Author(s) 2019

\begin{abstract}
Background Approximately 1-2\% of patients with non-small-cell lung cancer (NSCLC) harbor ROS1 rearrangements. Crizotinib, an oral small-molecule tyrosine kinase inhibitor (TKI) that targets anaplastic lymphoma kinase (ALK), MET, and ROS1, has shown marked antitumor activity in patients with ROS1-positive advanced NSCLC.

Objective Our objective was to analyze the efficacy and safety of crizotinib treatment in Chinese patients with advanced NSCLC with ROS1 rearrangement in real-world clinical practice.

Methods We included 35 patients with ROS1-positive NSCLC in this retrospective analysis. All received crizotinib $250 \mathrm{mg}$ twice daily between March 2016 and April 2018 at the Fudan University Shanghai Cancer Center. All had histologically or cytologically confirmed locally advanced or metastatic NSCLC with ROS1 rearrangements, which were identified by fluorescence in situ hybridization, reverse transcriptase polymerase chain reaction, or next-generation sequencing. The main outcome measures were progression-free survival (PFS), overall survival (OS), objective response rate (ORR), disease control rate (DCR), and adverse events.

Results The median age of the patients was 51.0 years; 23 (65.7\%) were female and 28 (80.0\%) were never smokers. All were diagnosed as having adenocarcinoma; eight patients (22.9\%) had brain metastases at baseline. The ORR and DCR were $71.4 \%$ and $94.3 \%$, respectively. The estimated median PFS was 11.0 months (95\% confidence interval [CI] 7.8-14.2). The estimated median OS was 41.0 months (95\% CI 22.5-59.5). Elevated transaminases (54.3\%), vision disorder (25.7\%), elevated blood creatinine (22.9\%), diarrhea (20.0\%), and vomiting (20.0\%) were the most commonly reported adverse effects. Conclusion Crizotinib was effective and well tolerated in Chinese patients with ROS1-positive advanced NSCLC in realworld clinical practice. The progression sites and patterns, as well as treatments after first disease progression on crizotinib were diverse. Crizotinib beyond progressive disease and local therapy after failure of crizotinib treatment were feasible and effective in clinical practice.
\end{abstract}

\section{Introduction}

Lung cancer remains the leading cause of cancer-related deaths worldwide, and non-small-cell lung cancer (NSCLC) accounts for approximately $85 \%$ of all lung cancers [1]. In the past decade, the treatment of NSCLC has undergone tremendous changes based on newly characterized key molecular alterations that drive lung carcinogenesis. The development of epidermal growth factor receptor (EGFR)-targeted tyrosine kinase inhibitors (TKIs) and anaplastic lymphoma

Chang Liu and Hui Yu contributed equally to this work.

Jialei Wang

luwangjialei@126.com

Extended author information available on the last page of the article

\section{Key Points}

ROS1 fusions are rare genetic abnormalities in non-smallcell lung cancer (NSCLC), and the small-molecule inhibitor crizotinib is an established treatment option for this disease.

Knowledge of disease progression patterns and treatments after progression on crizotinib in ROSI-positive NSCLC is currently limited.

This retrospective real-world study of 35 Chinese patients with ROS1-positive advanced NSCLC showed crizotinib to be effective and well-tolerated and that crizotinib beyond progression and local therapy after failure of crizotinib treatment were feasible and effective options in clinical practice. 
kinase (ALK)-targeted TKIs represent examples of individualized treatments for advanced NSCLC [2].

The c-ros oncogene 1 (ROS1) is also a molecular target in lung cancer. It encodes for an orphan receptor tyrosine kinase of the insulin receptor family and is related to $A L K$ and leukocyte receptor tyrosine kinase [3]. In 2007, chromosomal rearrangements involving the $\mathrm{ROS} 1$ gene, leading to fusions of the ROSI tyrosine kinase domain with one of several partner proteins, were reported $[4,5]$. Since then, oncogenic ROS1 rearrangements have become an established therapeutic target in lung cancer. Bergethon et al. [6] identified ROS1 rearrangements in 18 of 1073 patients (1.7\%) with NSCLC using fluorescence in situ hybridization (FISH), and suggested that ROS1 rearrangements define a unique molecular subset of NSCLC with distinct clinical characteristics, including younger patients (median age approximately 50 years) and never smokers, similar to those observed in patients with $A L K$-rearranged NSCLC.

Crizotinib, a multitargeted mesenchymal-epithelial transition/hepatocyte growth factor receptor (MET)/ALK/ROS1 inhibitor, has demonstrated remarkable efficacy in advanced ROS1-rearranged NSCLC and has consequently received approval from the US FDA and the European Medicines Agency (EMA) in 2016. This approval was based on efficacy and safety data from the expansion cohort of a phase I study (PROFILE 1001), which demonstrated an objective response rate (ORR) of $72 \%$ and a median progression-free survival (mPFS) of 19.2 months in patients with advanced ROS1-rearranged NSCLC [7].

Approximately $1-2 \%$ of patients with NSCLC harbor ROS1 rearrangements [4]. However, the incidence is slightly higher in East Asian populations, which report a frequency of 2-3\% [8]. A phase II, open-label, single-arm, multicenter trial assessing the efficacy and safety of crizotinib in a cohort of 127 East Asian patients with ROS1-positive advanced NSCLC (study OO1201) reported an ORR of $71.7 \%$ and an mPFS of 15.9 months [9]. Thus, crizotinib was approved in China for ROS1-positive NSCLC in September 2017 based on the results of study OO1201. Our current retrospective analysis aimed to analyze the efficacy and safety of crizotinib treatment in Chinese patients with advanced NSCLC with ROS1 rearrangement in real-world clinical practice at the Fudan University Shanghai Cancer Center.

\section{Patients and Methods}

\subsection{Patients}

In total, 35 patients (1.9\% of all screened patients) with NSCLC with ROS1 rearrangement were treated with crizotinib from March 2016 to April 2018 at the Fudan University Shanghai Cancer Center. All patients were histologically or cytologically diagnosed with locally advanced or metastatic NSCLC. All patients routinely had magnetic resonance brain imaging examinations at baseline. Positivity for ROSI rearrangements was determined using FISH, reverse transcriptase polymerase chain reaction (RT-PCR), or next-generation sequencing (NGS). The Vysis LSI ROS1 (Tel) SpectrumOrange Probe and LSI ROS1 (Cen) SpectrumGreen Probe (Abbott Molecular) were used for FISH testing. The AmoyDX and the ArcherDx FusionPlex ${ }^{\mathrm{TM}}$ panel were used for RT-PCR and NGS testing, respectively. We retrospectively collected clinical data and treatment outcomes from the patients' medical histories. Clinical stage was assigned according to the eighth edition of the tumor/node/metastasis (TNM) staging system.

This study was approved by the institutional review board of the Fudan University Shanghai Cancer Center. Informed consent was obtained from all patients.

\subsection{Treatment}

Patients were treated with oral crizotinib $250 \mathrm{mg}$ twice daily. The dosage could be reduced to $200 \mathrm{mg}$ twice daily, or permanently discontinued if adverse events (AEs) occurred.

\subsection{Efficacy and Safety Evaluation}

Efficacy was assessed by determining PFS, overall survival (OS), ORR, and the disease control rate (DCR). PFS was defined as the time from initiation of crizotinib therapy to the first disease progression on crizotinib or death. Patients alive without progression at the time of analysis were censored at their last follow-up. PFS2 was defined as the time from the first disease progression on crizotinib to the second disease progression or death, or to the next line of systemic therapy following crizotinib. OS was defined as the time from the firstline treatment of NSCLC with crizotinib to death. DCR was defined as the percentage of patients with a complete response $(\mathrm{CR})$, partial response (PR), and stable disease (SD), whereas ORR was defined as the percentage with CRs and PRs. Tumor response was initially assessed after 1 month of crizotinib therapy and every 2 months thereafter using the Response Evaluation Criteria In Solid Tumors (RECIST, version 1.1). Responses were defined as the best response from the start of treatment until disease progression.

AEs were assessed every month according to the National Cancer Institute's Common Terminology Criteria for Adverse Events (CTCAE), version 4.0.

\subsection{Statistical Analysis}

Data were summarized as frequencies and percentages for categorical variables and as median (range) for continuous variables. PFS and OS were estimated with the Kaplan-Meier 
method, along with hazard ratios. All outcome measures were calculated with $95 \%$ confidence intervals (CIs), which were estimated using the Cox proportional hazard model.

Exploratory univariate analyses were performed with a log-rank test for the following variables: sex, age, smoking history, number of metastatic sites (0-2 vs. $\geq 3$ ), liver/lung/ bone/brain metastases, line of crizotinib therapy ( $1 \mathrm{vs} . \geq 2$ ), clinical stage (IV vs. III), and the ROS1 detection method. Variables with a $p$ value $<0.2$ in the univariate analysis were included in a multivariate analysis using Cox multivariate models.

The significance level of statistical tests was set at $p<0.05$. All expressed $\mathrm{p}$-values and CIs were two-tailed. AEs were summarized using percentages and frequency counts. All statistical analyses were conducted using SPSS ${ }^{\circledR}$ Statistics version 24 (IBM; Armonk, NY, USA).

\section{Results}

\subsection{Patient Characteristics}

A total of 35 patients with ROS1-rearranged NSCLC were treated with crizotinib at the Fudan University Shanghai Cancer Center between March 2016 and April 2018. None of the patients had participated in a clinical trial. Their baseline characteristics at the initiation of crizotinib therapy are shown in Table 1 . The patients' median age was 51.0 years (range 26-82), and the majority were aged $<65$ years $(31 / 35$ [88.6\%]). There was a higher proportion of females (23/35 [65.7\%]) than males, and of never smokers (28/35 [80.0\%]) than former or current smokers. All patients were diagnosed as having adenocarcinoma; 30 $(85.7 \%)$ had stage IV disease at baseline and six $(17.1 \%)$ had postoperative recurrent disease. All 35 patients had an Eastern Cooperative Oncology Group performance status (ECOG PS) of 0-2.

Most patients (30/35 [85.7\%]) had distant metastasis detected at a routine examination before initiation of crizotinib treatment. The most common metastatic sites were bone $(40.0 \%)$, pleura $(34.3 \%)$, supraclavicular lymph node $(28.6 \%)$, lung $(22.9 \%)$, and brain $(22.9 \%)$. Crizotinib was used as first-line treatment in $17(48.6 \%)$ patients, as second-line treatment in $11(34.1 \%)$, and as third-line or later treatment in seven $(20.0 \%)$. In total, 18 patients received crizotinib as second- or later-line therapy; 15 of these patients $(15 / 18$ [83.3\%]) previously received pemetrexed combined with platinum chemotherapy followed by pemetrexed maintenance therapy, two of whom also received bevacizumab both as first-line chemotherapy and as maintenance therapy. Three other patients $(3 / 18$ [16.7\%]) received non-pemetrexed regimens plus platinum as first-line chemotherapy. Second-line or later-line chemotherapy regimens included docetaxel, paclitaxel, pemetrexed monotherapy, or regimens used in clinical trials. Six patients received radiotherapy before crizotinib, including one who received radiotherapy for a brain lesion, one for a bone lesion, and three for chest lesions. One patient received postoperative adjuvant radiotherapy.

ROS 1 rearrangements were determined using FISH (18/35 [51.4\%]), RT-PCR (15/35 [42.9\%]), or NGS (2/35 [5.7\%]). ROS1 fusion partners were identified in 12 patients (12/35 [34.3\%]). The most frequent ROS1 fusion partner was cluster of differentiation (CD)74 (CD74-E6; ROS1-E34), which was identified in eight patients (8/12 [66.6\%]). EZR (EZR-E10; ROS1-E34) was observed in two patients $(2 / 12$ [16.7\%]), and SDC4 (SDC4-E2; ROS1-E33) and SLC34A2 (SLC34A2-E4; ROS1-E32/E34) were observed in one patient $(1 / 12[8.3 \%])$, respectively.

\subsection{Efficacy}

Tumor responses are shown in Table 2. In total, 25 patients (71.4\%) achieved a PR and eight $(22.9 \%)$ had SD, resulting in an ORR of $71.4 \%$ (95\% CI 56.2-86.6) and a DCR of $94.3 \%$ (95\% CI 86.5-102.1). Two patients (5.7\%) who reported progressive disease (PD) as the best response had disease progression after 1 month of crizotinib treatment. In 17 patients who received crizotinib as first-line therapy, the ORR was $82.4 \%$, and the DCR was $100 \%$. In 18 patients treated with crizotinib as second- or later-line therapy, the ORR was $61.1 \%$, and the DCR was $88.9 \%$. The differences in ORRs and DCRs between patients treated with crizotinib as first-line therapy or later-line therapy were not statistically significant.

At the cutoff date (1 January 2019), 19 (54.3\%) patients were still being treated with crizotinib. The estimated median PFS was 11.0 months (95\% CI 7.8-14.2; Fig. 1), and $12(34.3 \%)$ patients were still in follow-up for PFS. The estimated median OS was 41.0 months $(95 \%$ CI 22.5-59.5; Fig. 2), and 23 patients (65.7\%) were still alive. In 17 patients treated with crizotinib as first-line therapy, the estimated median PFS was 13.0 months $(95 \%$ CI 6.5-19.5) compared with 8.0 months (95\% CI 3.8-12.2) in 18 patients treated with crizotinib as second- or laterline therapy; however, the difference was not statistically significant.

Several factors were analyzed for their ability to predict the PFS with crizotinib (Table 3$)$, including sex $(p=0.630)$, age ( $\geq 51$ vs. $<51$ years; $p=0.081)$, smoking history $(p=0.902)$, brain metastasis $(p=0.130)$, bone metastasis $(p=0.819)$, three or more metastatic sites $(p=0.737)$, treatment line (first- vs. second-line or later; $p=0.215$ ), and the ROS 1 detection method ( $p=0.004)$. The multivariate analysis identified only the ROS1 detection method as a significant factor predicting PFS $(p=0.046)$. 
Table 1 Baseline patient characteristics $(n=35)$

\begin{tabular}{|c|c|}
\hline Characteristic & No. of patients $(\%)^{\mathrm{a}}$ \\
\hline \multicolumn{2}{|l|}{ Age, years } \\
\hline Mean & 49.8 \\
\hline Median & 51.0 \\
\hline Range & $26-82$ \\
\hline \multicolumn{2}{|l|}{ Age group, years } \\
\hline$<65$ & $31(88.6)$ \\
\hline$\geq 65$ & $4(11.4)$ \\
\hline \multicolumn{2}{|l|}{ Sex } \\
\hline Male & $12(34.3)$ \\
\hline Female & $23(65.7)$ \\
\hline \multicolumn{2}{|l|}{ Smoking history } \\
\hline Never smoker & $28(80.0)$ \\
\hline Former or current smoker & $6(17.1)$ \\
\hline Unknown & $1(2.9)$ \\
\hline \multicolumn{2}{|l|}{ Histology } \\
\hline Adenocarcinoma & $35(100.0)$ \\
\hline \multicolumn{2}{|l|}{ ECOG PS at baseline } \\
\hline 0 & $1(2.8)$ \\
\hline 1 & $31(88.6)$ \\
\hline 2 & $3(8.6)$ \\
\hline \multicolumn{2}{|l|}{ Stage at baseline } \\
\hline IIIA & $2(5.7)$ \\
\hline IIIB & $3(8.6)$ \\
\hline IV & $30(85.7)$ \\
\hline M1a & $10(28.6)$ \\
\hline M1b & $1(2.9)$ \\
\hline M1c & $19(54.3)$ \\
\hline \multicolumn{2}{|l|}{ Brain metastases at baseline } \\
\hline Yes & $8(22.9)$ \\
\hline \multicolumn{2}{|l|}{ Metastatic sites at baseline } \\
\hline Lung & $8(22.9)$ \\
\hline Brain & $8(22.9)$ \\
\hline Bone & $14(40.0)$ \\
\hline Liver & $3(8.6)$ \\
\hline Adrenal gland & $2(5.7)$ \\
\hline Supraclavicular lymph node & $10(28.6)$ \\
\hline Pleural & $12(34.3)$ \\
\hline Others & $8(22.9)$ \\
\hline \multicolumn{2}{|l|}{ No. of metastatic sites } \\
\hline 0 & $5(14.3)$ \\
\hline 1 & $16(45.7)$ \\
\hline 2 & $7(20.0)$ \\
\hline$\geq 3$ & $7(20.0)$ \\
\hline \multicolumn{2}{|l|}{ ROS1 detection method } \\
\hline FISH & $18(51.4)$ \\
\hline RT-PCR & $15(42.9)$ \\
\hline NGS & $2(5.7)$ \\
\hline \multicolumn{2}{|l|}{ Lines of crizotinib therapy } \\
\hline 1 & $17(48.6)$ \\
\hline 2 & $11(31.4)$ \\
\hline$\geq 3$ & $7(20.0)$ \\
\hline
\end{tabular}

ECOG PS Eastern Cooperative Oncology Group performance status, FISH fluorescent in situ hybridization, NGS next-generation sequenc-
Table 1 (continued)

ing, $R T-P C R$ reverse transcriptase polymerase chain reaction

${ }^{\mathrm{a}}$ Unless otherwise stated

\subsection{Progression Patterns and Sequential Treatments}

The progression patterns of 21 patients who experienced disease progression are shown in Table 4 . The two patients who achieved PD as the best response were excluded from this analysis. The progression sites were the brain (10/21 [47.6\%]), lung (4/21 [19.0\%]), bone (2/21 [9.5\%]), liver (2/11 [9.5\%]), supraclavicular lymph node (1/21 [4.8\%]), pleura $(1 / 21[4.8 \%])$, and subcutaneous nodules $(1 / 21$ [4.8\%]); ten patients (47.6\%) developed PD in new lesions, whereas 11 patients $(52.4 \%)$ developed regrowth of previous lesions. Of the ten patients who experienced disease progression in the brain, six (60\%) had brain metastasis at baseline. Of the 11 patients who experienced disease progression at other sites, one (9.1\%) had brain metastasis at baseline. Patients with brain progression were more likely to have brain metastasis at baseline $(p=0.024)$.

Among all patients experiencing disease progression, $13(61.9 \%)$ continued crizotinib beyond PD (CBPD) for $>3$ weeks or until death (median 25 weeks), three (14.3\%) switched to lorlatinib, one (4.8\%) switched to brigatinib, one $(4.8 \%)$ received anlotinib, and the other three $(14.3 \%)$ received best supportive care after discontinuing crizotinib. Nine patients received local therapy after disease progression; seven received radiotherapy for brain disease, one received radiotherapy for subcutaneous nodules, and one received radiofrequency ablation for liver metastases. Two of these nine patients discontinued crizotinib therapy. The estimated median PFS2 was 21 weeks (95\% CI 6.8-35.2) for all 21 patients. The estimated median PFS2 was 25.0 weeks (95\% CI 1.4-48.6) in 13 patients who received CBPD, compared with 21.0 weeks (95\% CI 0.0-51.8) in eight patients who did not receive CBPD; however, the difference was not statistically significant $(p=0.386)$. Seven patients who received both CBPD and local therapy had a numerically longer PFS2 time ( 82.0 weeks) than the other 14 patients ( 21.0 weeks), although the difference was not statistically significant $(p=0.272)$.

\subsection{Safety}

Elevated transaminases (19/35 [54.3\%]), vision disorder (9/35 [25.7\%]), elevated blood creatinine (8/35 [22.9\%]), diarrhea (7/35 [20.0\%]), and vomiting (7/35 [20.0\%]) were the most commonly reported crizotinib-related adverse 
Table 2 Tumor responses

\begin{tabular}{llll}
\hline Responses & All patients $(n=35)$ & First-line crizotinib therapy $(n=17)$ & $\begin{array}{l}\text { Second-line or later- } \\
\text { line crizotinib therapy } \\
(n=18)\end{array}$ \\
\hline CR & $0(0.0)$ & 0 & 0 \\
PR & $25(71.4)$ & 14 & 11 \\
SD & $8(22.9)$ & 3 & 5 \\
PD & $2(5.7)$ & 0 & 2 \\
ORR & $71.4 \%(95 \%$ CI 56.2-86.6) & $82.4 \% *$ & $61.1 \%$ \\
DCR & $94.3 \%(95 \%$ CI $86.5-102.1)$ & $100 \% * *$ & $88.9 \%$ \\
\hline
\end{tabular}

Data are presented as $n$ or $\%$ and $95 \%$ CI

$C I$ confidence interval, $C R$ complete response, $D C R$ disease control rate, $O R R$ objective response rate, $P D$ progressive disease, $P R$ partial response, $S D$ stable disease

${ }^{*} p=0.264$ vs. second-line or later crizotinib therapy

$* * p=0.486$ vs. second-line or later crizotinib therapy

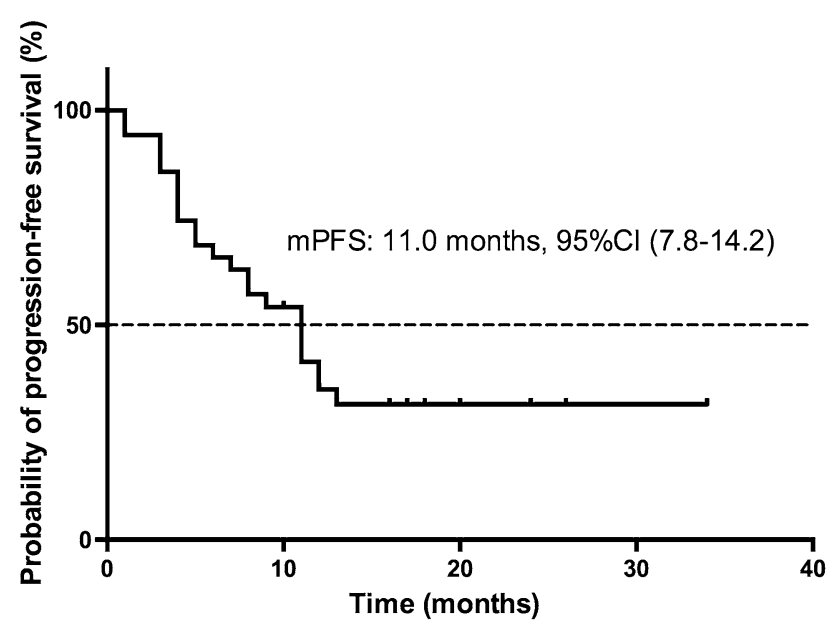

Fig. 1 Kaplan-Meier curve of progression-free survival of all patients $(n=35)$. $C I$ confidence interval, $m P F S$ median progressionfree survival

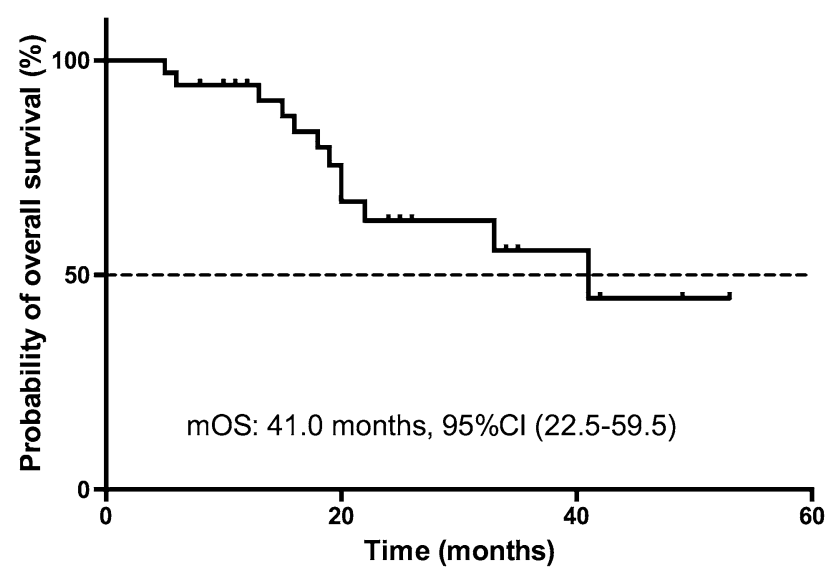

Fig. 2 Kaplan-Meier curve of overall survival of all patients $(n=35)$. $C I$ confidence interval, $m O S$ median overall survival effects (Table 5). Grade 3 events related to crizotinib were reported in only four patients (11.4\%), including grade 3 elevated transaminases in two patients, and grade 3 anemia and neutropenia in one patient. No unexpected AEs were observed. Three patients (8.6\%) discontinued crizotinib because of treatment-related AEs, one with grade 3 elevated transaminases and two with grade 2 vomiting.

\section{Discussion}

In the phase I PROFILE 1001 study, 50 patients with ROS1rearranged NSCLC who were treated with crizotinib (14\% as first-line therapy) achieved an ORR of $72 \%$ and a median PFS of 19.2 months [7]. On the basis of the efficacy and safety demonstrated in this study, crizotinib was granted full approval for the treatment of advanced ROS1-rearranged NSCLC by the FDA and the EMA in 2016 [7]. In a French phase II study [10] and in the EUROS1 retrospective analysis [11], the median PFS with crizotinib therapy for ROS1rearranged NSCLC was 9-10 months, although both these studies enrolled only approximately 30 patients. The largest study of crizotinib conducted to date, an East Asian phase II study (OO1201), achieved a median PFS of 15.9 months among 127 patients with ROS1-rearranged NSCLC and an ORR of $71.7 \%$ [9], which led to the subsequent approval of crizotinib for patients with NSCLC with ROS1 rearrangement in Japan, China, and Korea [9]. In this study, 18.9\% of patients received crizotinib as first-line therapy.

Our retrospective study analyzed the efficacy and safety of crizotinib in real-world clinical practice in Chinese patients, as few real-world studies have offered data on the efficacy and safety of crizotinib in Chinese patients with ROS1-rearrangement NSCLC (except for Chinese individuals in the published clinical trials). We demonstrated that 
Table 3 Exploratory analysis of the ability of various factors to predict progression-free survival with crizotinib therapy $(n=35)$

\begin{tabular}{lll}
\hline Variable & Univariable analysis $(p$ value $)$ & Multivariable analysis \\
& & Hazard ratio (95\% CI) \\
\hline Sex (male vs. female) & 0.630 & $0.366(0.093-1.444)$ \\
Age (<65 vs. $\geq 65$ years) & 0.130 & \\
Smoking history (yes vs. no) & 0.902 & $1.752(0.624-4.916)$ \\
Brain metastasis (yes vs. no) & 0.130 & \\
Bone metastasis (yes vs. no) & 0.819 & \\
Treatment line (first vs. others) & 0.215 & \\
Stage (IV vs. III) & 0.736 & \\
Metastasis ( $\geq 4$ vs. $<4$ sites) & 0.424 & \\
Metastasis ( $\geq 3$ vs. $<3$ sites) & 0.737 & \\
Metastasis ( $\geq 2$ vs. $<2$ sites) & 0.515 & \\
Metastasis (yes vs. no) & 0.736 & \\
Age $(\geq 51$ vs. $<51$ years) & 0.081 & \\
$R O S 1$ detection method & 0.004 & \\
\hline
\end{tabular}

CI confidence interval

Table 4 Progression patterns in 21 patients

\begin{tabular}{|c|c|c|c|c|c|c|c|}
\hline Patient no. & Sex & Response & PFS (months) & Progressive sites & Progression pattern & Treatment beyond progression & PFS2 (weeks) \\
\hline 1 & $\mathrm{~F}$ & PR & 3 & Bone & New lesion & CBPD & 65 \\
\hline 2 & M & SD & 3 & Liver & New lesion & $\begin{array}{l}\mathrm{CBPD}+\text { radiofrequency abla- } \\
\text { tion }\end{array}$ & 8 \\
\hline 3 & $\mathrm{~F}$ & SD & 13 & Pleura & New lesion & Lorlatinib & 25 \\
\hline 4 & M & PR & 4 & Brain & New lesion & CBPD + WBRT & 5 \\
\hline 5 & $\mathrm{~F}$ & PR & 11 & Brain & Regrowth & $\mathrm{RT}$ (brain) + CBPD & 60 \\
\hline 6 & M & $\mathrm{PR}$ & 4 & Brain & Regrowth & CBPD + WBRT & 82 \\
\hline 7 & $\mathrm{~F}$ & SD & 7 & Lung & Regrowth & CBPD & 25 \\
\hline 8 & $\mathrm{~F}$ & SD & 11 & Supraclavicular lymph node & New lesion & BSC & 52 \\
\hline 9 & $\mathrm{~F}$ & SD & 4 & Lung & Regrowth & $\mathrm{BSC}$ & 61 \\
\hline 10 & M & $\mathrm{PR}$ & 12 & Brain & Regrowth & CBPD & 4 \\
\hline 11 & M & SD & 12 & Brain & New lesion & $\begin{array}{l}\text { CBPD + WBRT + radiosur- } \\
\text { gery + ceritinib }\end{array}$ & 15 \\
\hline 12 & $\mathrm{~F}$ & PR & 5 & Brain & Regrowth & Lorlatinib & 21 \\
\hline 13 & $\mathrm{~F}$ & PR & 4 & Brain & New lesion & $\mathrm{CBPD}+\mathrm{RT}$ (brain) & 15 \\
\hline 14 & M & $\mathrm{PR}$ & 9 & Liver & New lesion & Lorlatinib & 0 \\
\hline 15 & $\mathrm{~F}$ & $\mathrm{PR}$ & 11 & Brain & New lesion & CBPD & 4 \\
\hline 16 & $\mathrm{~F}$ & PR & 11 & Lung & Regrowth & Brigatinib & 9 \\
\hline 17 & $\mathrm{~F}$ & SD & 8 & Brain & New lesion & $\mathrm{CBPD}+\mathrm{SBRT}$ & 10 \\
\hline 18 & $\mathrm{~F}$ & PR & 5 & Brain & Regrowth & $\mathrm{BSC}+\mathrm{RT}$ (brain) & 4 \\
\hline 19 & $\mathrm{~F}$ & PR & 8 & Bone & Regrowth & CBPD & 16 \\
\hline 20 & M & PR & 6 & Lung & Regrowth & CBPD & 4 \\
\hline 21 & $\mathrm{~F}$ & PR & 3 & SC nodules & Regrowth & Anlotinib + RT & 9 \\
\hline
\end{tabular}

$B S C$ best supportive care, $C B P D$ crizotinib beyond progressive disease, $F$ female, $M$ male, $P F S$ progression-free survival, $P F S 2$ time from the first disease progression on crizotinib to the second disease progression or death or to the next line of systemic therapy following crizotinib, $P R$ partial response, $R T$ radiotherapy, $S B R T$ stereotactic body radiation therapy, $S C$ subcutaneous, $S D$ stable disease, $W B R T$ whole-brain radiotherapy

crizotinib was effective in Chinese patients with ROS1positive advanced NSCLC, achieving an ORR of $71.4 \%$, a median PFS of 11.0 months (with 12 [34.3\%] patients still in follow-up for PFS) and a median OS of 41.0 months, with 23 patients $(65.7 \%)$ still alive. The median PFS with crizotinib therapy in our study was shorter than that reported in the 
Table 5 Adverse events reported $(n=35)$

\begin{tabular}{lcc}
\hline Adverse events & All grades & Grade 3 \\
\hline Any & $33(94.3)$ & $4(11.4)$ \\
Elevated transaminases & $19(54.3)$ & $2(5.7)$ \\
Vision disorder & $9(25.7)$ & \\
Elevated blood creatinine & $8(22.9)$ & \\
Vomiting & $7(20.0)$ & \\
Diarrhea & $7(20.0)$ & $1(2.9)$ \\
Anemia & $6(17.1)$ & \\
Fatigue & $6(17.1)$ & $1(2.9)$ \\
Edema & $5(14.3)$ & \\
Neutropenia & $5(14.3)$ & \\
Nausea & $4(11.4)$ & \\
Decreased appetite & $4(11.4)$ & \\
Leukopenia & $4(11.4)$ & \\
Constipation & $4(11.4)$ & \\
Thrombocytopenia & $3(8.6)$ & \\
Rash & $2(5.7)$ & \\
Dysgeusia & $2(5.7)$ & \\
\hline
\end{tabular}

Data are presented as $n(\%)$

PROFILE 1001 and OO1201 studies. This may reflect the inclusion of non-trial patients, limited sample sizes, and differences in patient demographics among the studies. However, the PFS values reported in the French phase II study and the EUROS1 study were comparable to those achieved in our study. Because 23 patients $(65.7 \%)$ were still alive, the OS data in our study were not mature. However, this is one of the few studies to produce OS data to date.

Several factors were explored for their ability to predict PFS with crizotinib therapy. However, the clinical efficacy of crizotinib might be irrespective of age, sex, smoking history, the presence of brain metastases at baseline, or line of crizotinib treatment, which is consistent with the findings of the OO1201 study. Responses were achieved in patients independently of prior lines of therapy, which indicates that crizotinib is beneficial in both first- and later-line settings. The patients' characteristics in our study were consistent with those of the China cohort of the O01201 study, including a median age of 51.0 years in our study compared with 49.5 years, $65.7 \%$ female compared with $54.1 \%$, and $100 \%$ adenocarcinoma compared with $95.9 \%$ in the OO1201 study.

The safety profile of crizotinib in our study was consistent with that reported in previous studies of crizotinib [7, 9, 12-14]. No unexpected AEs were observed. Crizotinibrelated AEs occurred in $94.3 \%$ of patients in our study. However, most AEs reported in our study were of grade 1 or 2 severity, and no grade 4 or higher crizotinib-related AEs were observed, which indicates that crizotinib is welltolerated in Chinese patients with ROS1-positive advanced NSCLC in real-world clinical practice. The most commonly reported crizotinib-related AEs in the OO1201 study [9] were elevated transaminases $(55.1 \%)$, vision disorder $(48.0 \%)$, nausea $(40.9 \%)$, diarrhea $(38.6 \%)$, and vomiting (32.3\%). The incidence and severity of AEs in our study were comparable to those in the O01201 study [9]. The AEs attributed to crizotinib were manageable with dosing interruptions or reductions, with a low rate of permanent treatment discontinuations due to crizotinib-related AEs.

Very few studies currently focus on progression patterns with crizotinib and sequential treatments after crizotinib failure in patients with ROS1-positive advanced NSCLC. Among the 21 patients who experienced disease progression in our study, the most common progression site was the brain (10/21 [47.6\%]), which is consistent with known data regarding poor crizotinib penetrance to the brain. Of the ten patients who experienced disease progression in the brain, six $(60 \%)$ had brain metastasis at baseline. Of the 11 patients who experienced disease progression at other sites, one $(9.1 \%)$ had brain metastasis at baseline. We postulated that patients with brain progression were more likely to have brain metastasis at baseline $(p=0.024)$. Poor penetration of crizotinib in the central nervous system (CNS) may account for both the short PFS and the high CNS progression rate. Furthermore, treatments beyond disease progression were diverse. The estimated median PFS2 was 21 weeks for all 21 patients experiencing PD after crizotinib. The estimated median PFS2 of 25.0 weeks in 13 patients who received CBPD was numerically longer than that of 21.0 weeks in eight patients who did not receive CBPD $(p=0.386)$. Seven patients who received both CBPD and local therapy had a longer PFS2 time of 82.0 weeks than the other 14 patients (21.0 weeks), although the differences were not statistically significant $(p=0.272)$. Despite the small sample size and the short follow-up time, we can infer that CBPD and local therapy after failure of crizotinib treatment were feasible and effective in clinical practice. Similarly, the prospective ASPIRATION study [15] supported the efficacy of first-line erlotinib therapy in Asian patients with EGFR mutation-positive NSCLC, and the feasibility of continuing erlotinib therapy beyond PD. Yang et al. [16] found that patients with local progression could benefit from continuation of EGFR-TKIs as systemic treatment plus local intervention after EGFR-TKI failure in clinical practice. In terms of failure after ALK-TKIs, Ou et al. [17] found that continuing $A L K$ inhibition with crizotinib after PD may provide a survival benefit for patients with advanced $A L K$-positive NSCLC. As radiotherapy can control brain tumors and improve CNS symptoms rapidly, to some extent compensating for poor penetration of crizotinib in the CNS, Hong et al. [18] found that continuation of both crizotinib and local therapy may contribute to disease control in patients with $A L K$-positive NSCLC 
and CNS progression during crizotinib treatment. Thus, our study highlights the need for further verification of the effectiveness of CBPD and local therapy after failure of crizotinib treatment, especially for locoregional progression in patients with ROS1-positive NSCLC in clinical practice.

This study is significant for several reasons. First, it provided first-hand real-world data on the efficacy of crizotinib in patients with advanced NSCLC with ROSI rearrangement. As ROS1-positive NSCLC is rare, it is difficult to perform large-scale, randomized, controlled, phase III clinical studies. Second, the safety profile noted in our study suggested that crizotinib is well-tolerated in Chinese patients with ROS1-positive advanced NSCLC in real-world clinical practice. In addition, we compared the safety profile between our study and the OO1201 study, which will help oncologists gain a better understanding of the possible adverse effects of crizotinib in real-world clinical practice. Third, we obtained information on disease progression at different sites in patients receiving crizotinib therapy and on treatment beyond disease progression in patients with ROS1 rearrangement NSCLC in real-world clinical practice. In the 21 patients who experienced disease progression, both the progression lesions and treatments beyond disease progression were diverse.

Our study has several limitations. First, as it was a single-center retrospective study with a relatively small sample size, possible information bias could have affected our outcomes. Second, the short follow-up time means the OS data were immature. Third, positivity for ROS1 rearrangements in our study was determined in about half our patients using the FISH detection method (18/35 [51.4\%]). Although the break-apart FISH assay is the only assay clinically approved by the FDA to detect ROS1-rearranged NSCLC, it has both advantages and disadvantages. FISH can be performed even if the exact fusion partner is unknown, as it has the potential to identify all fusions for ROSI in NSCLC and other solid tumors. On the other hand, the FISH assay cannot identify exact fusion partners, which can be confirmed by subsequent sequencing of the RT-PCR assay [19]. ROS1 fusion partners were identified in only 12 patients $(34.3 \%)$ in our study. The most frequent ROS1 fusion partner was CD74 (CD74-E6; ROS1-E34), which was identified in eight patients $(8 / 12$ [66.6\%]). When fusion partners are unknown, the effects of different fusion partners on the efficacy of crizotinib and on drug resistance cannot be analyzed, and this is the focus of current research on crizotinib resistance mechanisms in ROS1-rearranged NSCLC. Recently, one study that evaluated the roles of ROS 1 fusion partners on the treatment response found that patients with non-CD74 ROS1-positive NSCLC were less likely to have brain metastases and to have a trend towards an improved PFS [20].
In conclusion, this study showed that crizotinib was effective and well-tolerated in Chinese patients with ROS1-positive advanced NSCLC in real-world clinical practice and that progression sites and patterns, and treatments beyond disease progression after crizotinib were diverse. CBPD and local therapy after failure of crizotinib treatment were feasible and effective in clinical practice.

Acknowledgements Editorial assistance with the manuscript was provided by Content Ed Net, Shanghai Co. Ltd.

Author contributions JW designed the study, collected data and verified its integrity, and helped write the manuscript. CL and HY were responsible for statistical analysis and writing the manuscript. JC, HC, $\mathrm{YL}, \mathrm{WZ}, \mathrm{KZ}, \mathrm{ZZ}, \mathrm{SS}$, and MF were responsible for collecting and verifying the integrity of the data. All authors critically reviewed the manuscript, and all approved the final version submitted for publication.

\section{Compliance with Ethical Standards}

Funding No external funding was used in the preparation of this manuscript.

Conflict of interest Chang Liu, Hui Yu, Jianhua Chang, Haiquan Chen, Yuan Li, Weixin Zhao, Kuaile Zhao, Zhengfei Zhu, Si Sun, Min Fan, Jialei Wang have no conflicts of interest that are directly relevant to the content of this article.

Open Access This article is distributed under the terms of the Creative Commons Attribution-NonCommercial 4.0 International License (http://creativecommons.org/licenses/by-nc/4.0/), which permits any noncommercial use, distribution, and reproduction in any medium, provided you give appropriate credit to the original author(s) and the source, provide a link to the Creative Commons license, and indicate if changes were made.

\section{References}

1. Sateia HF, Choi Y, Stewart RW, et al. Screening for lung cancer. Semin Oncol. 2017;44:74-82.

2. Sgambato A, Casaluce F, Maione P, et al. Targeted therapies in non-small cell lung cancer: a focus on ALK/ROS1 tyrosine kinase inhibitors. Expert Rev Anticancer Ther. 2018;18:71-80.

3. Acquaviva J, Wong R, Charest A. The multifaceted roles of the receptor tyrosine kinase ROS in development and cancer. Biochim Biophys Acta. 2009; 1795:37-52.

4. Davies KD, Doebele RC. Molecular pathways: ROS1 fusion proteins in cancer. Clin Cancer Res. 2013;19:4040-5.

5. Rikova K, Guo A, Zeng Q, et al. Global survey of phosphotyrosine signaling identifies oncogenic kinases in lung cancer. Cell. 2007;131:1190-203.

6. Bergethon K, Shaw AT, Ou SH, et al. ROS1 rearrangements define a unique molecular class of lung cancers. J Clin Oncol. 2012;30:863-70.

7. Shaw AT, Ou SH, Bang YJ, et al. Crizotinib in ROS1-rearranged non-small-cell lung cancer. N Engl J Med. 2014;371:1963-71.

8. Chen YF, Hsieh MS, Wu SG, et al. Clinical and the prognostic characteristics of lung adenocarcinoma patients with ROS1 fusion in comparison with other driver mutations in East Asian populations. J Thorac Oncol. 2014;9:1171-9. 
9. Wu YL, Yang JC, Kim DW, et al. Phase II study of crizotinib in East Asian Patients With ROS1-positive advanced non-small-cell lung cancer. J Clin Oncol. 2018;36:1405-11.

10. Moro-Sibilot D, Faivre L, Zalcman G, et al. Crizotinib in patients with advanced ROS1-rearranged non-small cell lung cancer (NSCLC). Preliminary results of the ACSé phase II trial. J Clin Oncol. 2015;33(15):8065.

11. Mazieres J, Zalcman G, Crino L, et al. Crizotinib therapy for advanced lung adenocarcinoma and a ROS1 rearrangement: results from the EUROS1 cohort. J Clin Oncol. 2015;33:992-9.

12. Shaw AT, Kim DW, Nakagawa K, et al. Crizotinib versus chemotherapy in advanced ALK-positive lung cancer. N Engl J Med. 2013;368:2385-94.

13. Solomon BJ, Mok T, Kim DW, et al. First-line crizotinib versus chemotherapy in ALK-positive lung cancer. N Engl J Med. 2014;371:2167-77.

14. Camidge DR, Bang YJ, Kwak EL, et al. Activity and safety of crizotinib in patients with ALK-positive non-small-cell lung cancer: updated results from a phase 1 study. Lancet Oncol. 2012;13:1011-9.

15. Park K, Yu CJ, Kim SW, et al. First-line erlotinib therapy until and beyond response evaluation criteria in solid tumors progression in
Asian patients with epidermal growth factor receptor mutationpositive non-small-cell lung cancer: the ASPIRATION Study. JAMA Oncol. 2016;2:305-12.

16. Yang JJ, Chen HJ, Yan HH, et al. Clinical modes of EGFR tyrosine kinase inhibitor failure and subsequent management in advanced non-small cell lung cancer. Lung Cancer. 2013;79:33-9.

17. Ou SH, Jänne PA, Bartlett $\mathrm{CH}$, et al. Clinical benefit of continuing ALK inhibition with crizotinib beyond initial disease progression in patients with advanced ALK-positive NSCLC. Ann Oncol. 2014;25:415-22.

18. Hong X, Chen Q, Ding L, et al. Clinical benefit of continuing crizotinib therapy after initial disease progression in Chinese patients with advanced ALK-rearranged non-small-cell lung cancer. Oncotarget. 2017;8:41631-40.

19. Cao B, Wei P, Liu Z, et al. Detection of lung adenocarcinoma with ROS1 rearrangement by IHC, FISH, and RT-PCR and analysis of its clinicopathologic features. Onco Targets Ther. 2016;9:131-8.

20. Li Z, Shen L, Ding D, et al. Efficacy of crizotinib among different types of ROS1 fusion partners in patients with ROS1-rearranged non-small-cell lung cancer. J Thorac Oncol. 2018;13:987-95.

\section{Affiliations}

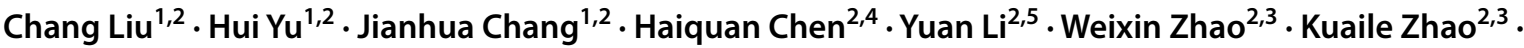 Zhengfei Zhu ${ }^{2,3} \cdot$ Si Sun $^{1,2} \cdot$ Min Fan $^{2,3} \cdot$ Jialei Wang ${ }^{1,2}$ (1)}

Chang Liu

liuchangfudan@163.com

Hui Yu

yhui30@ hotmail.com

Jianhua Chang

changjianhua@163.com

Haiquan Chen

hqchen1@yahoo.com

Yuan Li

lumoxuan2009@163.com

Weixin Zhao

zwx19@126.com

Kuaile Zhao

kuaile_z@sina.com

Zhengfei Zhu

zfeizhu@aliyun.com
Si Sun

sunsi123sun@aliyun.com

Min Fan

fanmin_zlyy@sina.com

1 Department of Medical Oncology, Fudan University Shanghai Cancer Center, Shanghai 200032, China

2 Department of Oncology, Shanghai Medical College, Fudan University, No. 270 Dong-An Road, Shanghai 200032, China

3 Department of Radiotherapy, Fudan University Shanghai Cancer Center, Shanghai 200032, China

4 Department of Thoracic Surgery, Fudan University Shanghai Cancer Center, Shanghai 20032, China

5 Department of Pathology, Fudan University Shanghai Cancer Center, Shanghai 200032, China 